\section{PROGNOSTIC AND PREDICTIVE VALUE OF PRE- TREATMENT T-CELL RECEPTORS (TCR) REPERTOIRE IN NON-SMALL CELL LUNG CANCER (NSCLC) PATIENTS TREATED WITH SINGLE AGENT IMMUNOTHERAPY}

${ }^{1}$ Afaf Abed*, ${ }^{1}$ Elin Gray, ${ }^{2}$ Michael Millward. ${ }^{1}$ Edith Cowan University, Joondalup, Australia; ${ }^{2}$ Linear clinical research, Perth, Australia

Background TCR repertoire plays a key role on the orchestration of the immune response. In particular, reduced pre-treatment Shannon diversity, increase clonality and increase convergence of TCRs have been suggested to reflect clonal expansion of antigen-specific T-cells in the tumour microenvironment. These are thought to be correlated with better response rate, improved progression free survival (PFS) and overall survival (OS). Here we aim to explore the above TCR repertoire features in peripheral blood of NSCLC patients (with PDL1 $\geq 50 \%$ ) treated with single agent pembrolizumab in the first line setting; and correlate them with overall response rate (ORR), PFS and OS.

Methods We prospectively collected baseline blood from 48 NSCLC patients treated with first line pembrolizumab. High quality DNA was extracted from white blood cells and used for TCR sequencing using the Oncomine TCR Beta-SR Assay (Thermo Fisher). TCR clonality and convergence were calculated for each individual and correlated with survival using Kaplan-Meier curves and survival statistics. Multivariate analysis was carried out controlling for other variable that may influence the association of TCR repertoire and outcomes such as age, sex, ECOG, smoking status and pre-treatment neutrophil to lymphocyte ratio (NLR).

Results Our data matured for 29 patients only with a followup of at least 6 months. We observed a trend towards increased pre-treatment TCR clonality in patients with objective response to pembrolizumab and statistically significant reduced Shannon diversity $(\mathrm{P}=0.042)$. Convergence did not seem to affect ORR in our cohort. Moreover, there was a significantly longer PFS in patients with reduced number of pretreatment clones $(\mathrm{HR}=0.40,95 \% \mathrm{CI} 0.14-1.17, \mathrm{P}=0.031)$, reduced Shannon diversity $(\mathrm{HR}=0.44,95 \% \mathrm{CI} 0.16-1.21, \mathrm{P}$ $=0.041)$, reduced Evenness $(\mathrm{HR}=0.31,95 \% \mathrm{CI} 0.11-0.94$, $\mathrm{P}=0.033)$ and elevated clonality $(\mathrm{HR}=3.18,95 \% \mathrm{CI} 1.06-$ $9.53, \mathrm{P}=0.033)$ (table 1). Reduced rather than increased convergence was correlated with a trend towards improved PFS. None of these parameters were statically significant in relation to OS (table 2).

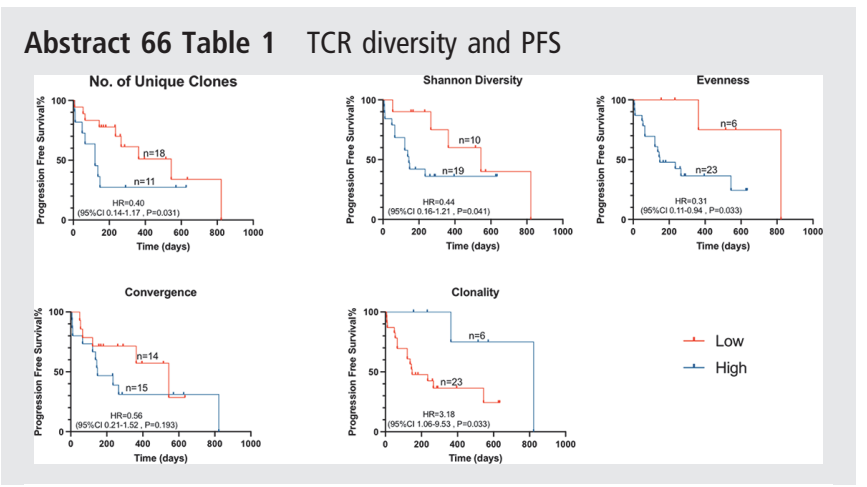

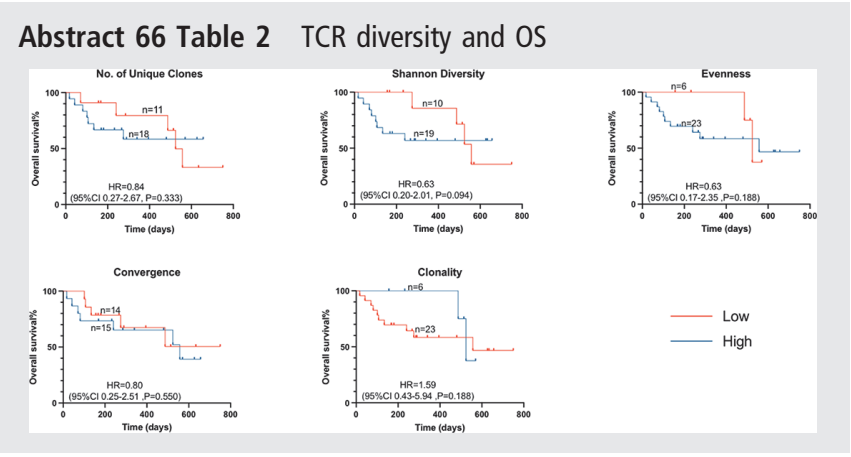

Conclusions Increased pre-treatment TCR clonality and reduced diversity are associated with improved ORR and PFS, but not OS in NSCLC patients with high PD-L1 treated with pembrolizumab monotherapy. Further maturation of this cohort will demonstrate whether the circulating pre-treatment TCR repertoire is a prognostic factor for immunecheckpoint inhibition.

Ethics Approval The proposed project has already received approval by the Human Research Ethics Committees and Research Governance at Fiona Stanley Hospital, Sir Charles Gairdner Hospital and Edith Cowan University [ECU (No. 18957) and SCGH (RGS0000003289)].

Consent Written informed consent was obtained from the patient for publication of this abstract and any accompanying images. A copy of the written consent is available for review by the Editor of this journal.

http://dx.doi.org/10.1136/jitc-2021-SITC2021.066 